\title{
Detection of Superior Markers for Polymerase Chain Reaction Diagnosis of Breast Cancer Micrometastasis in Sentinel Lymph Nodes
}

\author{
Shohreh Alizadeh Shargh ${ }^{1}$, Abolfazl Movafagh ${ }^{2 *}$, Nosratolah Zarghami ${ }^{3}$, Arezou \\ Sayad $^{2}$, Neda Mansouri ${ }^{2}$, Mohammad Taheri ${ }^{2}$, Atefeh Heidary Pour ${ }^{4}$, Mostafa \\ Iranpour ${ }^{2}$, Hamid Ghaedi ${ }^{2}$, Vahid Montazeri ${ }^{5}$, Nilofar Massoudi ${ }^{6}$, Mehrdad \\ Hashemi $^{7}$, SA Mortazavi-Tabatabaei ${ }^{8}$
}

\begin{abstract}
Breast cancer is the most prevalent type of cancer among women around the world, and mortality is primarily caused by micro-metastatic disease. The complex mechanisms of breast cancer invasion and metastasis are intrinsically related to the malignant cell type so that early detection of micro-metastases can help prolongation of survival for patient. The aim of the present research work was evaluation of the expression status of mammoglobin protein as a candidate molecular marker in the negative sentinel lymph node (SLN). Fifty tumor specimens, and 50 normal adjacent breast tissue samples from the same patients were selected on the basis of having more than $10 \%$ tumor content for RNA extraction from SLNs. Tumor samples and normal adjacent breast tissue were archived in the form of frozen fresh tissue in liquid nitrogen. Real-time PCR was performed on a Bioner life express gradient thermal cycler system. Mammoglobin gene overexpression in breast cancer metastasis was investigated. Single marker results were mammaglobin $66.7 \%$ and CK19 50.0\%, with 58.3\% for the two in combination. Due to improved outcome with at least 3 genes (83.3\%), it seems, triple marker evaluation will be most likely useful for detecting micro-metastases instead of studying separate genes.
\end{abstract}

Keywords: Breast cancer - micrometastases - sentinel lymph node - mammaglobin

Asian Pac J Cancer Prev, 17, Cancer Control in Western Asia Special Issue, 179-183

\section{Introduction}

Breast cancer is a prevalent tumor in women worldwide (Héry et al., 2008). Molecular diagnosis is recently proposed as a sensitive method combined with classic methods (Min et al., 1999). Detection of breast cancer is routinely performed by histological examination, but $30-35 \%$ of node negative patients are not diagnosed and may develop metastasis in the next 5 years after local treatment (Nemoto et al., 1980). The sensitivity of histological method for micro metastasis detection is so far low and about $20 \%$ (Nissan et al., 2006).

Sentinel lymph node (SLN) evaluation is a good predictor of axillary lymph node metastasis in breast cancer and SLN analysis during the first surgery, and can reduce the cost and risks of a second operation (Backus et al., 2005). Using reliable molecular diagnostic tests may help fill the gap between these subjects. Assessment of some genes that are involved in breast cancer metastasis may help accurate diagnosis, and comparative evaluation of these markers, and will provide an extremely sensitive method for detection of micrometastases for breast cancer micro metastasis. To fulfill this need, selection of an ideal marker is very important with high specificity and sensitivity, close to $100 \%$.

Mammoglobin is a member of uteroglobin/clara cell protein family (secretoglobins) (Klug et al., 2000) that is ideal for outcome of breast cancer metastasis evaluation in blood, lymph nodes, and bone marrow (Backus et al 2005). Mammoglobin is expressed in breast epithelial cells (O'Brien et al., 2002), and is overexpressed close to $70-80 \%$ of primary and metastatic breast cancers (Watson et al., 2009). In this study the expression status of mammoglobin protein is evaluated as a candidate molecular marker in negative sentinel lymph nodes using RT-PCR method.

${ }^{1}$ Department of Medical Laboratory Science, Midwifery-Nursing Institute, Islamic Azad University of Chalous Branch, Chalous, ${ }^{2}$ Department of Medical Genetics, Pediatric Neurology Research Center, ${ }^{6}$ Department of Anesthesiology, School of Medicine, ${ }^{8}$ Proteomics Research Center, School of Paramedical Sciences, Shahid Beheshti University of Medical Sciences, ${ }^{4}$ Department of Cell and Molecular Biology, Pharmaceutical Sciences Branch, ${ }^{7}$ Department of Genetic, Islamic Azad University, Tehran, ${ }^{5}$ Department of General Surgery, School of Medicine, Hematology and Oncology Research Center, ${ }^{3}$ Department of medical Biotechnology, Tabriz University of Medical Sciences, Tabriz, Iran.*For correspondence: Movafagh.a@sbmu.ac.ir 


\section{Materials and Methods}

The samples in this study were obtained from a biobank of Pathology department, Imam Reza referral and teaching hospital, Tabriz, Iran during May 2011- Jan 2012. The project was authorized and granted by Azad Islamic University of Chalous Branch- registration no: 123-06-1,388. This research and patients care were assigned on the basis of National/International breast cancer programs and approved according to official Iranian law and regulations by the Institutional Review Boards of each participating teaching and referral hospital. Also all patients staged supported by official American Joint Committee on Cancer (AJCC) according to the attending surgeon using correct medical history and histological data. Tumor samples were archived in the form of frozen fresh tissue in liquid nitrogen. Fifty sentinel lymph node samples and 50 normal breast tissue from the same patients were originally stored until the time of analysis. At the time of RNA extraction, patients were diagnosed with stage I or II in primary breast cancer with no distant metastasis, treated with mastectomy or lumpectomy plus axillary dissection, with or without postoperative radiation therapy and with or without adjuvant tamoxifen monotherapy. 50 tumor specimens, and 50 normal breast tissue specimens from the same patients were selected on the basis of having more than $10 \%$ tumor content for RNA extraction Sentinel lymph node.

\section{Sample preparation}

All patients were classified as coding groups and all private information was stored and saved secretly by authorized researcher of project.

30 sentinel lymph node RNA specimen available from the tumor bank of pathology department of medical multicenter cancer Imam Khomeini hospital, Tehran medical university were prepared. Also 10 breast ductal carcinomas and 1 benign breast tissue were procured from stored personal DNA bank of authors. This study aimed to the usefulness of qualitative measurement of mammoglobin protein, as a marker in lymph nodes. In this order, the negative (Hematoxylin/Eosin staining) lymph nodes are marked as case samples and the positive (metastatic samples or $\mathrm{H} / \mathrm{E}$ positive) breast cancer tissues are considered as control samples.

For this evaluation RNA quality was evaluated using Eppendorf spectrophotometry and beta-actin was used as housekeeping gene in PCR analysis (Muyal et al., 2009). RNA of lymph node and breast tissue samples were showed to be of less value if either of the housekeeping gene gave signals that was at least 3.5 cycles over the optimum of lymph node and other specimen checked. Low quality RNA samples were not found. There were 30 patients with same number lymph nodes included in the final data set from this study. The RNA was undergone to reverse transcription reaction to make a cDNA before performing RT-PCR. The obtained RNA was stored in form of separate $10 \mu \mathrm{l}$ in $-20^{\circ} \mathrm{C}$ until next processing. RNA was used in $4 \mu \mathrm{g}$ concentration, $26 \mathrm{ng} / \mu \mathrm{l}$ oligo dT (Biolab, New England) was added and incubated at $63^{\circ}$
$\mathrm{C}$ for 5 minutes and the product transferred on ice. Then in final $25 \mu \mathrm{l}$ reaction volume, included $1 \times$ protoscript

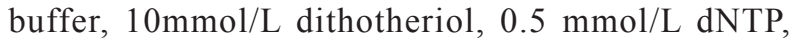
$10 \mathrm{U} / \mu 1 \mathrm{M}-\mathrm{MLV}$ (Moloney Murine Leukemia Virus) reverse transcriptase enzyme (Biolab, first strand cDNA synthesis kit) $0.25 \mathrm{U} / \mu \mathrm{l}$ RNase inhibitor (Biolab). This mix incubated in $37{ }^{\circ} \mathrm{C}$ for 1 hour and after that, incubated in $92^{\circ} \mathrm{C}$ for 6 minutes. The obtained product (cDNA) was diluted 1:5 with distilled water and stored at $-20^{\circ} \mathrm{C}$. Real-Time assay was conducted for mammoglobin marker and beta-2-macroglobinas housekeeping gene. Primers and probes are as follows: mammoglobin: AF015224 (Mikhitarian et al., 2008), for CK19, Y0050 (Leelawat et al., 2012), for MUC1 (Wreesmann et al., 2004), For MTDH1, NM_17881 (http://www.net/gene/gene), and about the beta- 2 macroglobin: AB021288 as housekeeping control gene with amplicone size around $88 \mathrm{bp}$ (Shrout et al., 2008). Real-time RT-PCR was performed on a Bioner life express gradient thermal cycler System (Waltham, MA). In each reaction, negative control was set up with a sample of water and reaction mix shown in Table 1.

\section{Results}

Analysis of mammoglobin expression level in breast cancer tissues, benign breast tissues and sentinel lymph nodes, showed that this marker was not found in benign breast tissues thus, the molecular of this marker has a $100 \%$ specificity.

Evaluating the outcome of single sequence figure, the four genes selected as markers, were investigated on lymph nodes of near 30 sentinel lymph node dissected patients.

The remarkable number of pathology-negative specimen shows most value of sign for several of the tested markers.

There was a significant relationship between tumor size and positive results of all markers in analyzed sentinel lymph nodes (Pearson chi-square test, $\mathrm{p}<0.01$ ). In evaluation of other tumor markers such as tumor's stage, grade, ER expression levels there was no significant variation with positive expression results of each genes (data not shown).

According to these findings, the highest single gene expression was of $66.7 \%$ for mammoglobin, followed by Ck19 50\% and MTDH, 26.7\% in Figure 1.

There was no significant relationship between tumor's stage and grade and positive markers results.

According to the availability of important expression of minimum 3 genes in $83.3 \%$ (40 of the 48 ) negative patients, it seems that triple marker evaluation of most prevalent expressed gene instead of separate gene, analysis will be more useful in metastasis prognosis exhibited in Figure 2. In statistical analysis of data, there were no significant difference in each studied gene alone but in combination, the significant variation was observed in pathologically negative sentinel lymph nodes in comparison with pathologically positive reported samples (10 pathologically positive samples used as negative control for marker expression) $(\mathrm{p}=0.01)$. Also as none of sentinel lymph node samples belong to pathologically positive samples, calculating of sensitivity 
Table 1: Cyber green assay and primer sequences.

\begin{tabular}{lcr}
\hline Gene & Type & sequence $\left(5^{\prime}\right.$-3') \\
\hline $\begin{array}{l}\text { Mammogl } \\
\text { obin }\end{array}$ & $\mathrm{F}$ & ATAGTAACCAGGCGTCTCGTGG \\
CK19 & $\mathrm{R}$ & CGGATGAAACTCTGAGCAATGT \\
& $\mathrm{F}$ & CATGAAAGCTGCCTTGGAAGA \\
MUC-1 & $\mathrm{R}$ & TGATTCTGCCGCTCACTATCAG \\
& $\mathrm{R}$ & ACCATCCTATGAGAGCGAGTACC \\
MTDH1 & $\mathrm{F}$ & TTACCACCATTACCTGCAGAAAC \\
& $\mathrm{R}$ & ATTCCAGCCTCCTCCATTGAC \\
$\begin{array}{l}\text { Beta-2 } \\
\text { macroglo }\end{array}$ & $\mathrm{F}$ & TGAGTGCTGTCTCCATGTTTGA \\
bin & $\mathrm{R}$ & TCTGCTCCCCACCTCTAAGTTG \\
\end{tabular}

$\mathrm{F}$, forward primer $\mathrm{R}$, reverse primer

and specificity was not possible.

\section{Performance of marker combinations}

In order the best and most reliable results, there is to measure the correct unit of 2 or more genes for coworker with histopathology. The better exon can have the correct the essential for basis of an intra-operative marker to find of metastasis in breast tumor sentinel lymph nodes. For this reason for single marker results (mammaglobin $66.7 \%$, CK19 50.0\%), most reliable marker combination also was calculated for most prevalent single genes in combination (mammaglobin/CK 19, 58.3\%), that shows the highest prevalence in positive results. Addition of a third marker did not complement MG/CK/MUC or MG/ $\mathrm{CK} / \mathrm{MTDH}$ in the negative samples. The negative results suggest that the majority of them, are due to unequal specimen for the dividing of test for PCR and oncology analysis, rather than from lack of expression of the single technic analyzed. These interpretation is partly helped by the fact that the some of tested negative primary samples, demonstrated substantial expression of mammaglobin and or/ CD19 after 4-5 months later (data not shown).

The combination of two genes (mammaglobin and CK19) is thought to be the best variety of reasons marker set for analysis. At first, the triple set for example additional of MUC1, has minimal positive results and not convincing ( 5 from 25 ), and for MTDH1 the positive results were weakly positive. Secondly, more of other gene to a multiple test would likely have a negative impact on manual quality. Furthermore, the less outcome expression of MUC1 and MTDH1 made them unsuitable targets of an intra-operative test and because the interest to increasing cycle numbers for obtaining positive results (ct threshold).

\section{Discussion}

Based on previous studies and in the light of our previous paper, there are few important genes that have a central role in lymphatic node metastasis in breast cancer and identified according to different methods such as microarray analysis (Tafreshi et al., 2012; Shargh et

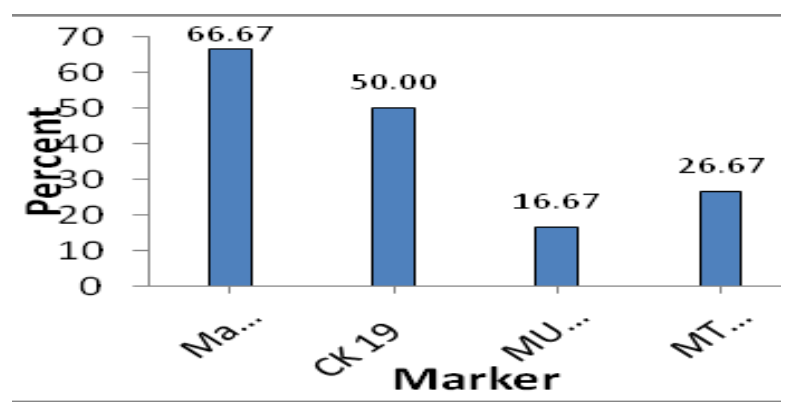

Figur 1. Prevalence of Positive Result of Four Marker in Diffrent Tumors Stage and Grade

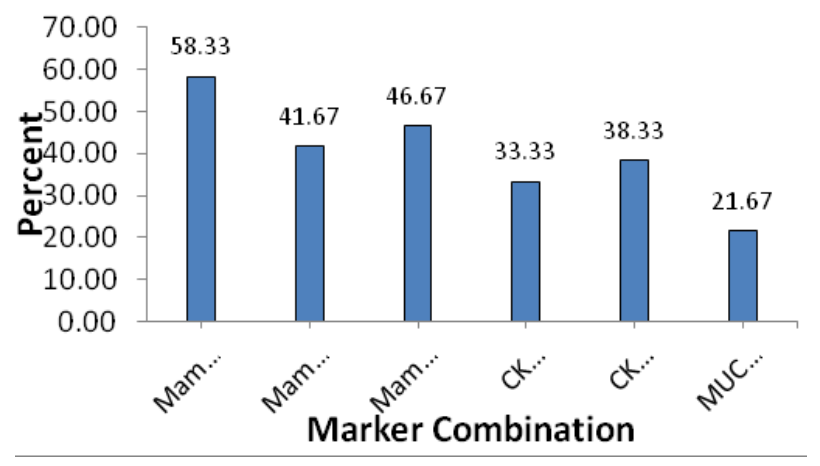

Figure 2. Comparison of Combinational Positive result for Metastatic Markers Positive Result in RT-PCR Analysis of Sentinel Lymph Nodes

\section{al., 2014).}

In the present study, the samples are set as molecular marker-positive, $\mathrm{H} \& \mathrm{E}$ negative from patients with some degree of micrometastasis that were not detected by standard pathology.

In this study, four of these genes used validation for finding of breast metastasis in SLN. The significant test and interesting individual of all metastasis cases with combination of these genes (markers) was evaluated in four and two marker sets. This investigation, also has reported the finding of a correct 2-gene outcome expression marker set for detection of medically important metastasis in breast sentinel lymph nodes. Among other markers, the 2 unique markers, recognize, mammaglobin and CK19 that has been published before as possibly ideal for multi-marker set of finding for occult metastasis in breast lymph nodes (Raynor et al., 2009).

(Viale et al., 20081) found that the multi-marker RT-PCR test recorded in $\% 6$ of SLNs in which metastases was not found by common pattern of histology as the metastases was lost as a result of the less-numbering of sampling (Viale et al., 20081). Actual volume of lymph node in present study was around the half of the node.

Moreover, the assay of fresh sentinel lymph node is more sensitive than the frozen part of specimen evaluation, which has been reported to range from $44 \%$ to $74 \%$ among different research work (Langer et al., 2009; Tanis et al., 
2001).

According to (Liu et al., 2010) in the evaluation 29 cases with positive results, out of which, 27 cases were positive for CK19 gene and 19patients for MG, 16 cases were positive for both CK19 and MG genes. BLN test positivity is correlated with higher stage for breast cancer with a rate of $71.4 \%$ (5.7) for stage such as T3 compared with $26.7 \%$ (24/90) for earlier T stages ( $\mathrm{P}=0.01)$, therefore, no association was detected between BLN assay positivity and other tumor performance (tumor type, histological grade, hormonal and HER2 status).

The advantage of RT-PCR assay in detection of micrometastasis is high sensitivity as it can detect only one copy of gene RNA and therefore the rate of false-positive results is reduced, although this high sensitivity may result to reducing specify. Combinational detection with multi-marker assay, is suitable for application in intraoperative assay to direct lymph node dissection and correct decision for performing it or not.

Finding the additional markers that are only in cancer cells and not as much as in advanced cancer cells, is the method that can be used for detection of micrometastasis. This suggested to be added in this combination and will a more reliable than two marker set for detection of micrometastasis in axillary sentinel lymph nodes.

\section{Acknowledgment}

This work was supported by deputy research administration, Shahid Beheshti University of Medical Sciences, Tehran, Iran. Also the result of this research work is the thesis work of Shohreh Alizadeh Shargh.

\section{Conflict of interest}

The authors have no financial relationships to disclose and no conflicts of interest to report.

\section{References}

Backus J, Laughlin T, Wang Y, et al (2005). Identification and characterization of optimal gene expression markers for detection of breast cancer metastasis. J Mol Diagn, 7, 327-36.

Darvish H, Heidari A, Hosseinkhani S, et al (2013). Biased homozygous haplotypes across the human caveolin 1 upstream purine complex in Parkinson's disease. J Mol Neurosci, 51, 389-93.

Emamalizadeh B, Movafagh A, Akbari M, et al, (2014). RIT2, a susceptibility gene for Parkinson's disease in Iranian population. Neurobiol Aging, 35, 27-8.

Héry C, Ferlay J, Boniol M, et al (2008). Quantification of changes in breast cancer incidence and mortality since 1990 in 35 countries with Caucasian-majority populations. Ann Oncol, 19, 1187-94.

Jamshidi J, Movafagh A, Emamalizadeh B, et al (2014). HLADRA is associated with Parkinson's disease in Iranian population. Int J Immunogenet, 41, 508-11.

Klug J, Beier HM, Bernard A, et al (2000). Uteroglobin/Clara cell 10-kDa family of proteins: nomenclature committee report. Ann N Y Acad Sci, 923, 348-54.

Langer I, Guller U, Berclaz G, et al (2009). Swiss Multicenter Study Group Sentinel Lymph Node in Breast Cancer. Accuracy of frozen section of sentinel lymph nodes: a prospective analysis of 659 breast cancer patients of the
Swiss multicenter study. Breast Cancer Res Treat, 113, 129-36.

Leelawat K, Narong S, Udomchaiprasertkul W, et al (2012). Prognostic relevance of circulating CK19 mRNA in advanced malignant biliary tract diseases. World $J$ Gastroenterol, 18, 175-81.

Liu YH, Xu FP, Liao N, et al (2010). Efficacy of intraoperative GeneSearch Breast Lymph Node (BLN). Assay for breast cancer metastasis detection in sentinel lymph node in Chinese patients. Cancer Sci, 101, 1920-4.

Mikhitarian K, Martin RH, Ruppel MB, et al (2008). Detection of mammaglobin mRNA in peripheral blood is associated with high grade breast cancer: interim results of a prospective cohort study. BMC Cancer, 8, 55-61.

Min CJ, Tafra L, Verbanac KM, et al (1998). Identification of superior markers for polymerase chain reaction detection of breast cancer metastases in sentinel lymph nodes. Cancer Res, 58, 4581-4.

Movafagh AZ, Pear Zadeh M, HajiseyedJavadi, et al (2008). Occurrence of congenital anomalies and genetic diseases in a population of Ghazvin province, Iran: A study of 33380 cases. Pak J Med Sci, 24, 80-5.

Muyal JP, Muyal V, Kaistha BP, et al (2009). Systemic comparison of RNA extraction techniques from frozen and fresh lung tissues: checkpoint towards gene expression studies. Ann Diagn Pathol, 4, 9-17.

Nemoto T, Vana J, Bedwani RN, et al (1980). Management and survival of female breast cancer: results of a national survey by the American College of Surgeons. Cancer, 45, 2917-24.

Nissan A, Jager D, Roystacher M, et al (2006). Multimarker RT-PCR assay for the detection of minimal residual disease in sentinel lymph nodes of breast cancer patients. $\mathrm{Br} J$ Cancer, 94, 681-85.

O'Brien N, Maguire TM, O'Donovan N, et al (2002). Mammaglobin a: a promising marker for breast cancer. Clin Chem, 48, 1362-64.

Raynor MP, Stephenson SA, Pittman KB, et al (2009). Identification of circulating tumour cells in early stage breast cancer patients using multi marker immunobead RT-PCR. $J$ Hematol Oncol, 5, 24-30.

Shargh SA, Sakizli M, Khalaj V, Movafagh A, et al (2014). Down regulation of E-cadherin expression in breast cancer by promoter hypermethylation and its relation with progression and prognosis of tumor. Med Oncol, 31, 250.

Shrout J, Yousefzadeh M, Dodd A, et al (2008). beta(2) macroglobulin mRNA expression levels are prognostic for lymph node metastasis in colorectal cancer patients. $\mathrm{Br} J$ Cancer, 98, 1999-2005.

Tafreshi NK, Bui MM, Bishop K, et al (2012). Noninvasive detection of breast cancer lymph node metastasis using carbonic anhydrases IX and XII targeted imaging probes. Clin Cancer Res, 18, 207-19.

Tanis PJ, Boom RP, Koops HS, et al (2001). Frozen section investigation of the sentinel lymph node in malignant melanoma and breast cancer. Ann Surg Oncol, 8, 222-6.

Viale G, Dell'Orto P, Biasi MO, et al (2008). Comparative evaluation of an extensive histopathologic examination and a real-time reverse-transcription-polymerase chain reaction assay for mammaglobin and cytokeratin 19 on axillary sentinel lymph nodes of breast carcinoma patients. Ann Surg, 247, 136-42.

Watson MA, Fleming TP (1996). Mammaglobin, a mammary-specific member of the uteroglobin gene family, is overexpressed in human breast cancer. Cancer Res, 56, $860-5$.

Wreesmann VB, Sieczka EM, Socci ND, et al (2004). Genome-wide profiling of papillary thyroid cancer identifies 
DOI:http://dx.doi.org/10.7314/APJCP.2016.17.S3.179

Detection of superior markers for Polymerase Chain Reaction Diagnostic of Breast Cancer Micrometastasis in Sentinel Lymph Nodes MUC1 as an independent prognostic marker. Cancer Res, 64, $3780-9$. 